\title{
Within-modality and cross-modality attentional blinks in a simple discrimination task
}

\author{
GRIT HEIN, ALICE PARR, and JOHN DUNCAN \\ MRC Cognition and Brain Sciences Unit, Cambridge, England
}

\begin{abstract}
Following up on studies of the "attentional blink," we studied interference between successive target stimuli in visual and auditory modalities. In each experiment, stimuli were two tones and four dots, simultaneously presented for 1,800 msec. Targets were brief intensity changes in either a tone or a dot. Subjects gave unspeeded responses. In four experiments, our results showed interference between targets in the same modality, but not across modalities. We conclude that, under our experimental conditions, restrictions in concurrent target identification are largely modality specific.
\end{abstract}

Attentional limits have been shown in many processing domains. For example, it is difficult to identify two simultaneous speech streams (Broadbent, 1958) or two separate objects in a visual display (Duncan, 1984). Subjectively, it is hard to divide attention between these stimuli. One interesting set of experiments concerns the "attentional blink." In these experiments, two target stimuli (T1 and T2) are presented with varying temporal separation. Both targets must be detected or identified. Even if targets themselves are very brief, attentional limits are shown by impaired processing of T2 up to a stimulus onset asynchrony (SOA) of $500 \mathrm{msec}$ or more (Pashler \& Badgio, 1987; Raymond, Shapiro, \& Arnell, 1992).

What sort of limit is shown by this impairment? One possibility is competition or conflict in modality-specific processing systems. Many physiological results, for example, show that concurrent visual stimuli compete for representation at multiple levels of the cortical visual system (Chelazzi, Duncan, Miller, \& Desimone, 1998; Desimone \& Duncan, 1995). More broadly, however, simultaneous tasks or processing operations can interfere for many reasons. Interference is often worst for very similar tasks, as one would expect if similar tasks share many local processing systems (Allport, 1980; Navon \& Gopher, 1979). Even if tasks are very different, however, some interference generally remains (Bourke, Duncan, \& Nimmo-Smith, 1996; Kahneman, 1973). Here, we investigate the specificity of interference reflected in the attentional blink. Specifically, we compare interference for targets in the same or in different sensory modalities (Treisman \& Davies, 1973).

Our starting point is a set of experiments reported by Duncan, Martens, and Ward (1997). On each trial, subjects were asked to identify two target words, with varying

This work was supported by the Human Frontier Science Program. Correspondence concerning this article should be addressed to J. Duncan, MRC Cognition and Brain Sciences Unit, 15 Chaucer Road, Cambridge CB2 2EF, England (e-mail: john.duncan@mrc-cbu.cam.ac.uk).
SOAs. In three different experiments, Duncan et al. studied cases in which both targets were auditory, both were visual, or there was one of each. A detailed consideration of these experiments introduces some of the questions and methods of the present work.

In the within-modality auditory experiment, each trial had two simultaneous speech streams, one spoken in a high voice and one in a low voice. Each stream consisted of repetitions of the nontarget syllable "guh," with a single target word embedded somewhere within it. The first target (T1) occurred unpredictably in either the high-voice or the low-voice stream; following an SOA of 125-1,375 msec, the second target (T2) occurred in the other stream. In the divided-attention condition, subjects listened to both streams and reported both target words. In focused-attention control conditions, subjects listened to just one voice or the other, and so identified only one target on each trial. Results are shown in Figure 1A. Accuracy rates for T1 and $\mathrm{T} 2$ are plotted, respectively, at negative and positive SOAs; data at -125 and $+125 \mathrm{msec}$, for example, show T1 and T2 accuracy, respectively, for a 125-msec SOA between them. Comparison of the divided-attention (open circles) and control (filled circles) data shows two results. First, divided attention was associated with an overall loss of accuracy across SOAs. Second, as in typical attentional blink studies, specific interference between T1 and T2 was shown by a further drop of accuracy at SOAs at least up to $375 \mathrm{msec}$.

The results of the within-modality visual experiment were similar (Figure 1B). Again, each trial had two target words, one presented unpredictably in a box either above or below fixation, and the other presented unpredictably in a box to the left or right. Targets were again presented in a stream of nontargets (rows of $x$ s flashing repeatedly in all four boxes). In focused-attention control conditions (attention to only the above/below positions, or attention to only the left/right positions), accuracy was high and independent of SOA. In the divided-attention condition, an overall loss in accuracy was again accompanied by specific T1-T2 interference at short SOAs. 

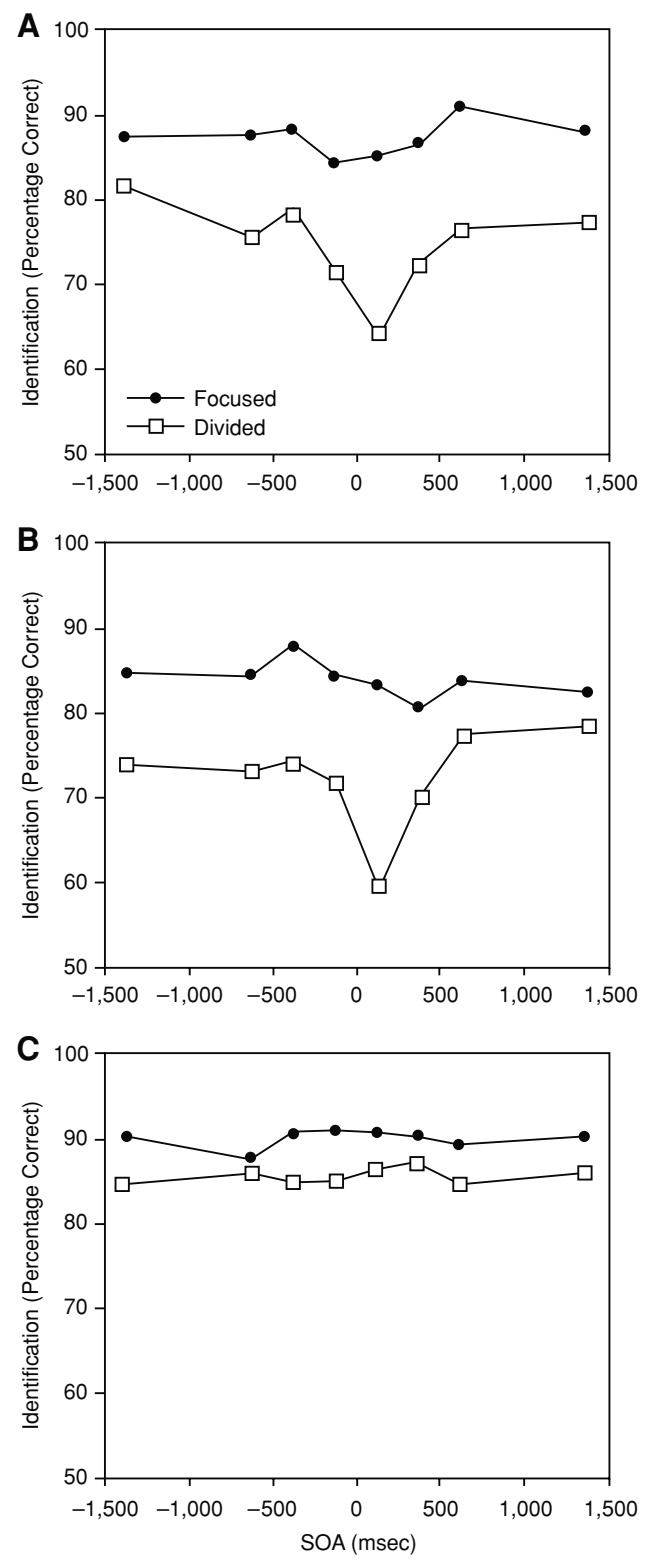

Figure 1. Results of Duncan et al. (1997). Group mean percentage of correct responses as a function of condition (focused or divided attention) and stimulus onset asynchrony (SOA). Positive SOAs reflect $T 2$ performance at each $S O A$ after $T 1$. Negative SOAs reflect T1 performance at each SOA backward from T2. (A) Auditory experiment. (B) Visual experiment. (C) Cross-modality experiment. Adapted with permission from Duncan et al. (1997).

In the cross-modality experiment, one auditory target (embedded in the high-voice stream) was accompanied by one visual target (presented in a left/right location). Again (Figure 1C), there was a general loss of accuracy in the divided-attention condition, but this time with no additional loss at short SOAs.

General interference in these tasks, not time-locked to target presentation, could be ascribed to many causes.
Divided-attention conditions, for example, require more complex task preparation (Pashler, 1994) and control (Logan, 1978). Interference time-locked to target presentation, however, suggests specific conflicts in concurrent target identification. The Duncan et al. (1997) results show much stronger conflict for targets in the same sensory modality.

A number of subsequent experiments have also examined the attentional blink within and between sensory modalities. As we have said, simultaneous activities can be affected by processing conflict at many different levels (Allport, 1980). As we might expect, accordingly, circumstances can certainly be found with significant interference, even across modalities. For example, cross-modal blinks can be strong if either or both targets require a speeded response (Jolicœur, 1999), especially if response selection demands are complex (Arnell \& Duncan, 2002). For unspeeded tasks, however, the cross-modal effect is usually weaker and less stable than its within-modality counterpart (Arnell \& Jenkins, 2004; Arnell \& Larson, 2002; Potter, Chun, Banks, \& Muckenhoupt, 1998; SotoFaraco \& Spence, 2002; for an exception, see Arnell \& Jolicœur, 1999). For unspeeded tasks, the results suggest that the major source of the attentional blink is withinmodality processing competition.

One limitation of previous work has been the rather narrow range of tasks investigated. For unspeeded identification tasks, stimuli have usually been symbols, such as letters, numbers, or words. Here, we ask what happens with simpler stimuli and discriminations. To compare withinand cross-modality blinks, we adapted materials from a previous cross-modal divided-attention study (Bonnel \& Hafter, 1998). In the Bonnel and Hafter experiments, subjects were presented with a simultaneous $500-\mathrm{Hz}$ tone (auditory pedestal) and a $3-\mathrm{cm}$ lighted circle (visual pedestal), each lasting for $900 \mathrm{msec} .500 \mathrm{msec}$ after stimulus onset, targets occurred in one modality (in focused-attention blocks) or in both modalities (in divided-attention blocks). Each target was a brief change of intensity - that is, a change in luminance of the circle or a change in volume of the tone. In the identification condition, which is of most interest here, subjects had to identify whether each intensity change was a decrement or an increment. In different divided-attention blocks, subjects were asked to vary the amount of attention they allocated to the auditory or the visual channel (e.g., 50\% auditory, 50\% visual; $80 \%$ auditory, $20 \%$ visual). Responses were unspeeded. The results showed a clear divided-attention decrement: Targets in each modality were identified best in focused-attention blocks, and progressively less well as the percentage of attention decreased. This finding suggests interference between processing of auditory and visual targets in a simple, unspeeded discrimination task.

In the present study, in four attentional blink experiments, we used targets like those used by Bonnel and Hafter (1998). The design of our experiments is based on the Duncan et al. (1997) study, with one important exception. Duncan et al. presented a pair of visual streams and a 
pair of auditory streams in the visual and auditory withinmodality experiments. The cross-modality experiment, on the contrary, had only one auditory stream and one visual stream. It could be argued that the difference in results between the within- and the cross-modality experiments was due to this difference in presentation conditions. According to several models, one function of focused attention is to protect a stimulus from sensory noise or interference produced by overlapping or nearby stimuli (Lu \& Dosher, 2000; Reynolds, Chelazzi, \& Desimone, 1999). Plausibly, using only one auditory and one visual stream in the cross-modal experiment could have reduced this sensory interference, and hence attentional sensitivity. To control for this possibility, we used concurrent stimulation within the visual and within the auditory modality in all withinand cross-modality experiments.
At the same time, our design allows us to assess effects of SOA for simple intensity discriminations. In the Bonnel and Hafter (1998) study, there was only a single SOA of 0 msec. Even in the cross-modal experiment of Duncan et al. (1997), at short SOAs there was certainly a performance difference between divided attention and focused attention (Figure 1C). As shown by other SOAs, however, this difference was not a specific consequence of identifying two stimuli close together in time. Examining a range of SOAs allows us to assess time-locked interference with stimuli like those that Bonnel and Hafter used.

\section{GENERAL METHOD}

In all experiments, stimuli were four dots and two tones (Figure 2), all presented simultaneously for $1,800 \mathrm{msec}$. Targets were brief in-

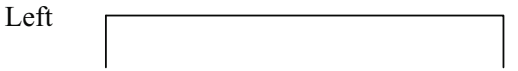

Horizontal

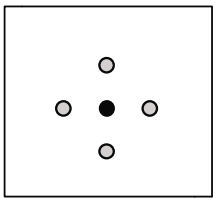

Right

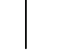

Above

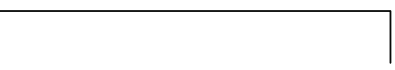

Vertical

Below
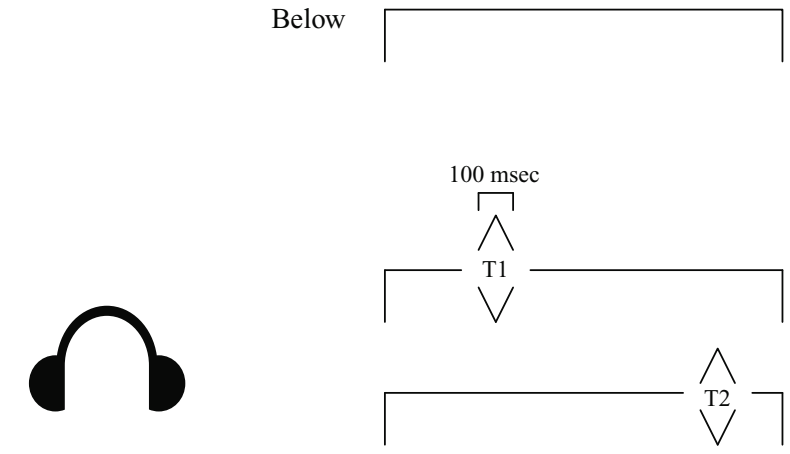

$1100 \mathrm{~Hz}$

$400 \mathrm{~Hz}$

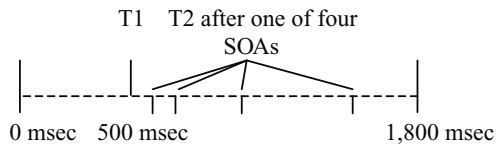

Figure 2. Example trial. Subjects heard a low tone $(400 \mathrm{~Hz}$, low channel) and a high tone $(1100 \mathrm{~Hz}$, high channel) and saw the dot display. For purposes of the design, the dot display was regarded as a "horizontal channel" (the dots to the left and right of the fixation dot) and a "vertical channel" (the dots above and below the fixation dot). The presentation of the tones and dots lasted $1,800 \mathrm{msec}$. $\mathrm{T} 1$, here an auditory target, was presented after $500 \mathrm{msec}$. T2 followed after one of four stimulus onset asynchronies (SOAs). Here, it is an auditory target, presented at the long SOA. Auditory targets were increments and decrements in the intensity of either the low or the high tone. Visual targets were increments or decrements in the intensity of one of the dots. When a target occurred on the horizontal channel, it was unpredictably to the left or right, and when a target occurred on the vertical channel, it was unpredictably above or below. 
creases or decreases in the intensity of a dot or tone. For purposes of the design, the four dots were considered to be organized into two pairs, acting as separate "channels." As in the experiments of Duncan et al. (1997), the "horizontal channel" consisted of the two dots to the left and right of fixation, whereas the "vertical channel" consisted of the two dots above and below fixation. When a horizontal target occurred, it was unpredictably to the left or right, and when a vertical target occurred, it was unpredictably above or below. This way, central fixation was always required, even when a subject was monitoring only one visual channel. When an auditory target occurred, it was either an intensity change of the high tone ("high channel") or low tone ("low channel").

Dots were presented $2.9^{\circ}$ from fixation, at a luminance of $42.7 \mathrm{~cd} /$ $\mathrm{m}^{2}$. Except in Experiment 4, each target was a 100-msec decrement or increment of luminance, decrements from $42.7 \mathrm{~cd} / \mathrm{m}^{2}$ to $7.7 \mathrm{~cd} / \mathrm{m}^{2}$, and increments from $42.7 \mathrm{~cd} / \mathrm{m}^{2}$ to $148.3 \mathrm{~cd} / \mathrm{m}^{2}$. These values were based on pilot tests with a single-channel identification task, aiming for approximately $80 \%$ correct responses. Tones were $400 \mathrm{~Hz}(74 \mathrm{~dB})$ in the left ear and $1100 \mathrm{~Hz}(80 \mathrm{~dB})$ in the right ear, presented over ATA-1118 headphones (Fujikon, Hong Kong). An auditory target was a 100 -msec ramped decrement or increment of the volume of the high or low tone. Each target ramped linearly up or down to maximum amplitude change at $50 \mathrm{msec}$, and then back to the original value at $100 \mathrm{msec}$. In Experiment 1, maximum amplitude change (amplitude change at $50 \mathrm{msec}$ ) was $100 \%$. Through an oversight, smaller changes (maximum $\pm 25 \%$ ) were used in other experiments, but any overall difference in performance was small compared with the very large differences always seen between individual subjects.

Although four dots and two tones were present on every trial of all experiments, for each experiment, only two channels were relevant. Each trial had one target on each of the relevant channels, in unpredictable order. Targets never occurred on the remaining, irrelevant channels. Each subject served in three conditions: two focused-attention control conditions, requiring attention to only one channel, and the divided-attention condition, requiring attention to both.

On each trial, tones and dots were initiated simultaneously by the subject pressing the space bar. T1 was presented $500 \mathrm{msec}$ after stimulus onset, unpredictably in one of the two relevant channels. Except in Experiment 4, T2 followed after an SOA of 100, 200, 500, or 1,000 msec, always in the other channel (Figure 2). As in the Bonnel and Hafter (1998) experiment, subjects had to identify each intensity change as an increment or decrement. Each response was a two-alternative forced choice, typed on the computer keyboard after the stimulus presentation ended. For each experiment, one pair of keys $(D$ and $F$ ) was used for "increment" and "decrement" responses for one relevant channel; another pair ( $J$ and $K$ ) was used for the other channel. In focused-attention conditions, subjects attended to only one channel, and gave a single response for the target in that channel, whether it occurred first or second. Under dividedattention conditions, subjects typed in one response for each channel, with no order constraint. Subjects were strongly encouraged to take their time when responding.

All experiments began with a familiarization phase, in which subjects were introduced to general task procedures and the discriminations to be made. This was followed by a number of 20 -trial practice blocks, usually 1 per condition, but with more for subjects who experienced particular difficulties. Data were then collected in six experimental blocks ( 64 trials each) that were organized into three pairs, one pair of blocks per condition. As far as possible, we counterbalanced the order of conditions across subjects. At the beginning of each block, a short instruction was presented in the middle of the screen, telling the subjects which channel(s) they had to attend to. Within a block, every combination of SOA and target order appeared equally often, in random order. Each experiment was conducted in a single session and lasted approximately $1.5 \mathrm{~h}$.
To prevent ceiling and floor effects, subjects were excluded if mean accuracy was $\geq 95 \%$ or $\leq 55 \%$, averaged across conditions and SOAs. In Experiments 3 and 4, this criterion was applied separately to auditory and visual data.

\section{EXPERIMENT 1}

In Experiment 1, we tested interference between targets in the auditory modality. Thus, relevant channels were the low tone and the high tone.

\section{Method}

This experiment had 19 paid subjects, ages 20-32 years. Two subjects were excluded for performance outside accuracy cutoffs (for 1 , accuracy was $>95 \%$; for the other, $<55 \%$ ). Three further subjects were excluded for failure to comply with instructions. Thus, 14 complete data sets were analyzed.

The response keys were $D$ and $F$ for the low tone and $J$ and $K$ for the high tone. $D$ and $J$ were labeled louder; $F$ and $K$ were labeled softer. Subjects were asked to fixate the fixation dot, to ignore visual stimuli, and to identify the auditory targets.

\section{Results and Discussion}

Figure 3 shows the average accuracy (percentage correct) depending on the interval between the targets (SOA) for the focused- and the divided-attention conditions. Performance scores for T1 and T2 were calculated independently of whether the response to the other target was correct. Mean accuracy scores were computed for each SOA for each subject and submitted to an ANOVA including condition (focused vs. divided) and SOA as withinsubjects variables.

As in the study of Duncan et al. (1997), the data showed an overall advantage for focused over divided attention. In divided attention, there was an additional performance decrement at short SOAs. Though strongest for T2, this decrement was also present for T1. In agreement with these conclusions, an ANOVA showed significant main effects of condition $[F(1,13)=10.5, p<.01]$ and SOA $[F(7,91)=2.9, p<.01]$, as well as a significant interaction $[F(7,91)=2.2, p<.005]$.

In agreement with several previous results (Duncan et al., 1997; Mondor, 1998; but see Potter et al., 1998), these data show a marked attentional blink when $\mathrm{T} 1$ and T2 are both presented in the auditory modality.

\section{EXPERIMENT 2}

In Experiment 2, we tested interference between targets in the visual modality. Thus, relevant channels were the horizontal and vertical.

\section{Method}

Seventeen paid subjects, ages $22-35$ years, participated in this experiment. Eight subjects were excluded for performance outside accuracy cutoffs (for $6,>95 \%$; for $2,<55 \%$ ). One further subject was excluded for failure to comply with instructions. Analyses were based on the remaining 8 .

Subjects were instructed to fixate the fixation dot and to identify changes of intensity in the vertical or horizontal dots, or both (see 


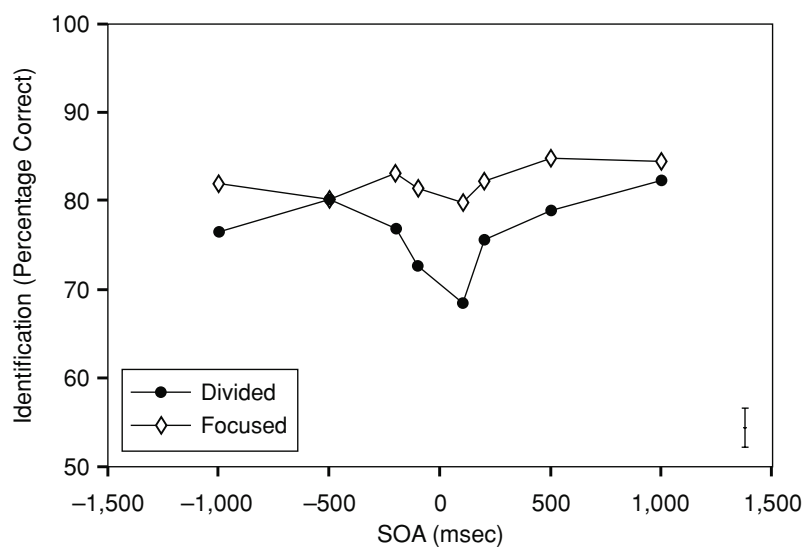

Figure 3. Experiment 1: Group mean percentage of correct responses to two auditory targets as a function of condition (focused or divided attention) and stimulus onset asynchrony (SOA). Positive SOAs reflect $\mathrm{T} 2$ performance at each $\mathrm{SOA}$ after $\mathrm{T} 1$. Negative SOAs reflect $\mathrm{T} 1$ performance at each SOA backward from $\mathrm{T} 2$. The error bar (bottom right) shows an estimate of \pm 1 standard deviation of the mean for each graph point, based on pooled within-subjects error terms.

Figure 2). The response keys for the horizontal channel were $D$ and $F$ on the keyboard; the response keys for the vertical channel were $J$ and $K$. $D$ and $J$ were labeled brighter, $F$ and $K$ were labeled dimmer.

\section{Results and Discussion}

Again, a general loss of accuracy in divided attention was accompanied by a clear attentional blink at short SOAs (Figure 4). An ANOVA showed significant main effects of condition $[F(1,7)=26.9, p<.01]$ and SOA $[F(7,49)=6.1, p<.001]$, as well as a significant interaction $[F(7,49)=3.1, p<.01]$. The results show the expected attentional blink when both $\mathrm{T} 1$ and $\mathrm{T} 2$ are visual.

\section{EXPERIMENT 3}

In Experiment 3, we turned to the cross-modality case. The high tone was the relevant auditory channel, and the horizontal was the relevant visual channel.

\section{Method}

Twenty subjects, ages $23-30$, participated in this experiment. One was excluded for failure to follow instructions. Auditory data were analyzed for 13 subjects, excluding 6 for performance outside accuracy cutoffs (for $3,>95 \%$; for $3,<55 \%$ ). Visual data were analyzed for 12 subjects, excluding 7 (all $>95 \%$ ).

As in the previous experiments, both auditory channels and both visual channels were presented simultaneously. Subjects had to identify targets in the horizontal visual channel and the $1100-\mathrm{Hz}$ tone. The response keys were $D$ and $F$ for the visual response and $J$ and $K$ for the auditory response. $D$ was labeled with brighter; $F$ with dimmer; $J$ with louder; $K$ with softer.

\section{Results and Discussion}

Results appear in Figures 5A (auditory targets) and 5B (visual targets). In agreement with the data of Duncan et al. (1997), the results suggest no hint of an attentional blink. Also in agreement with the earlier data, performance overall was better for focused attention, at least for auditory targets. ANOVAs with condition (focused attention/ divided attention) and SOA as within-subjects variables were conducted separately for auditory and visual targets. For auditory targets, condition was significant $[F(1,12)=$ $8.2, p<.02]$, reflecting overall better performance in focused attention. However, neither SOA $[F(7,84)=1.2$, $p>.3]$ nor SOA $\times$ condition $[F(7,84)<1]$ reached significance. For visual targets, none of the effects was significant [condition, $F(1,11)=1.3, p>.2$; SOA, $F(7,77)<1$; SOA $\times$ condition, $F(7,77)<1]$.

A further analysis compared blink sizes in Experiment 3 versus those in Experiments 1 and 2. To measure blink size, we used T2 data for SOAs of 100 and $200 \mathrm{msec}$. Blink size was calculated as the mean percentage correct for focused attention minus the mean percentage correct for divided attention. A $t$ test comparing auditory blink sizes in Experiments 3 and 1 showed a difference of borderline significance $[t(25)=1.53, p<.07$, one-tailed]. The corresponding test comparing visual blink sizes in Experiments 3 and 2 showed a highly significant difference $[t(18)=2.84$, $p<.01]$. Together, these results show a substantial overall difference in blink sizes for within-modality and crossmodality conditions.

\section{EXPERIMENT 4}

The results of our first three experiments, which used simple targets and discriminations, showed strong attentional blinks for targets in the same modality, but not for targets in different modalities. Most previous attentional blink experiments using symbolic stimuli have also shown that, at least for unspeeded tasks, interference is reduced

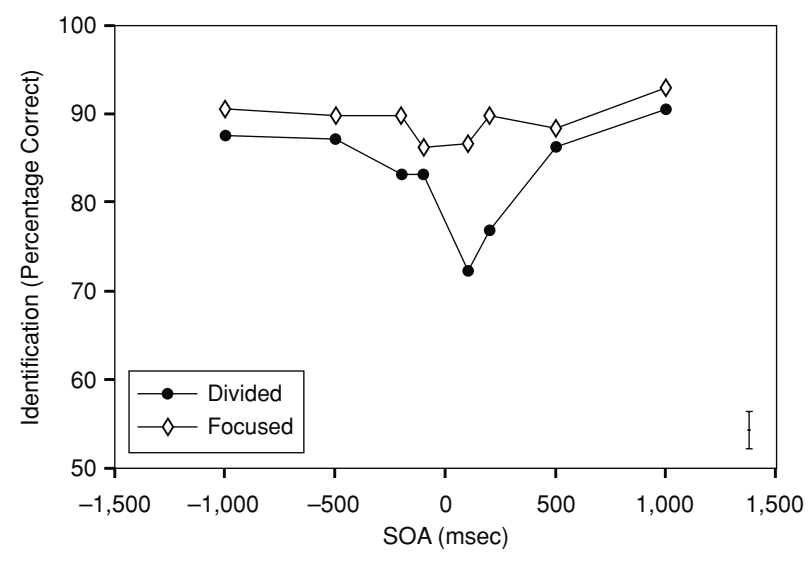

Figure 4. Experiment 2: Group mean percentage of correct responses to two visual targets as a function of condition (focused or divided attention) and stimulus onset asynchrony (SOA). Positive SOAs reflect T2 performance at each SOA after T1. Negative SOAs reflect $\mathrm{T} 1$ performance at each SOA backward from $\mathrm{T} 2$. The error bar (bottom right) shows an estimate of \pm 1 standard deviation of the mean for each graph point, based on pooled within-subjects error terms. 

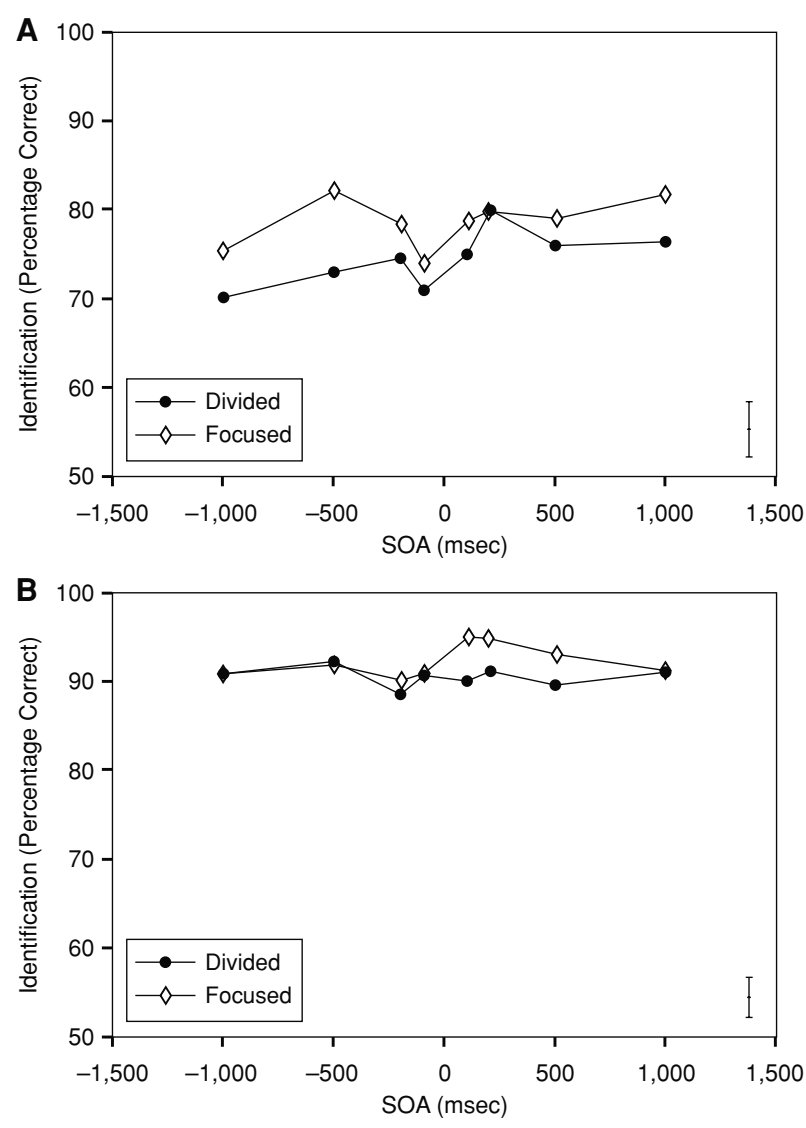

Figure 5. Experiment 3: Group mean percentage of correct responses as a function of condition (focused or divided attention) and stimulus onset asynchrony (SOA). Positive SOAs reflect T2 performance at each SOA after $T 1$. Negative SOAs reflect $T 1$ performance at each SOA backward from T2. (A) Auditory targets. (B) Visual targets. The error bars (bottom right of each panel) show estimates of \pm 1 standard deviation of the mean for each graph point, based on pooled within-subjects error terms.

or eliminated in the cross-modal case (Arnell \& Jenkins, 2004; Arnell \& Larson, 2002; Duncan et al., 1997; Potter et al., 1998; Soto-Faraco \& Spence, 2002).

Experiment 3 did show a general advantage for focused attention over divided attention, at least for auditory data. To this extent, our data resemble those of Bonnel and Hafter (1998), who also obtained a divided-attention decrement using only a single SOA of $0 \mathrm{msec}$. Along with the previous results of Duncan et al. (1997), however, our data suggest that this decrement is not specifically time-locked to target identification. As a further check on this conclusion, Experiment 4 included a condition with simultaneous auditory and visual targets. To reduce the problem of ceiling effects in visual data, we also reduced the duration of visual targets from 100 to $30 \mathrm{msec}$.

\section{Method}

Fourteen subjects, ages 23-28, participated in this experiment. Both auditory and visual data were analyzed for 11 subjects, in each case excluding 3 for performance outside accuracy cutoffs $(<55 \%)$. SOAs were $0,200,500$, and $1,000 \mathrm{msec}$, again occurring equally often. For nonzero SOAs, as before, there were equal numbers of trials with auditory T1/visual T2 and with visual T1/auditory T2. Visual target duration was reduced to $30 \mathrm{msec}$. Apart from these changes, the procedure was exactly the same as in Experiment 3.

\section{Results and Discussion}

Results are shown in Figure 6A (auditory targets) and 6B (visual targets). Just as in Experiment 3, there was a general trend for worse performance in the divided-attention condition, but no suggestion of additional decrement at short SOAs.

Mean percentages correct were submitted to ANOVAs with condition (focused attention vs. divided attention) and SOA as within-subjects variables. None of the variables reached significance for auditory targets [condition, $F(1,10)<1$; SOA, $F(6,60)=1.8, p>.1$; SOA $\times$ condition, $F(6,60)<1$ ] or for visual targets [condition, $F(1,10)<1$; SOA, $F(6,60)=1.4, p>.2$; SOA $\times$ condition, $F(6,60)=1.2, p>.3]$.

All in all, Experiment 4 replicated the results of Experiment 3. Even at the zero SOA, and with more difficult visual discriminations, there was still no time-locked interference between visual and auditory targets.

\section{GENERAL DISCUSSION}

In four experiments, we tested for restrictions of attentional capacity within and across the visual and auditory modalities. In particular, we studied attentional blinks produced by identification of two targets presented close together in time. Extending previous attentional blink studies, we used a simple discrimination, with concurrent stimulation within both modalities, and unspeeded responses. At short SOAs, our results showed large interference between targets in the same modality. On the contrary, there was no such effect if an auditory and a visual target had to be identified. Our last experiment confirmed this result even for simultaneous target presentation.

Although our main focus was on the attentional blink (i.e., interference time-locked to target identification), we frequently observed an additional, general dividedattention decrement seen across all SOAs. In withinmodality experiments, this SOA-independent decrement was added to the attentional blink. In cross-modality experiments, the blink was absent, but trends for a general divided-attention decrement remained. As we discussed earlier, there are many possible explanations for such general decrements in divided- compared with focusedattention blocks (see, e.g., Logan, 1978; Pashler, 1994). Our data suggest, however, that such general decrements should be clearly distinguished from specific conflicts in concurrent target identification.

Our choice of stimuli for these studies was suggested by the previous cross-modality study of Bonnel and Hafter (1998). Because those authors used only a single SOA of $0 \mathrm{msec}$, their results could not distinguish a general divided-attention decrement from specific interference 

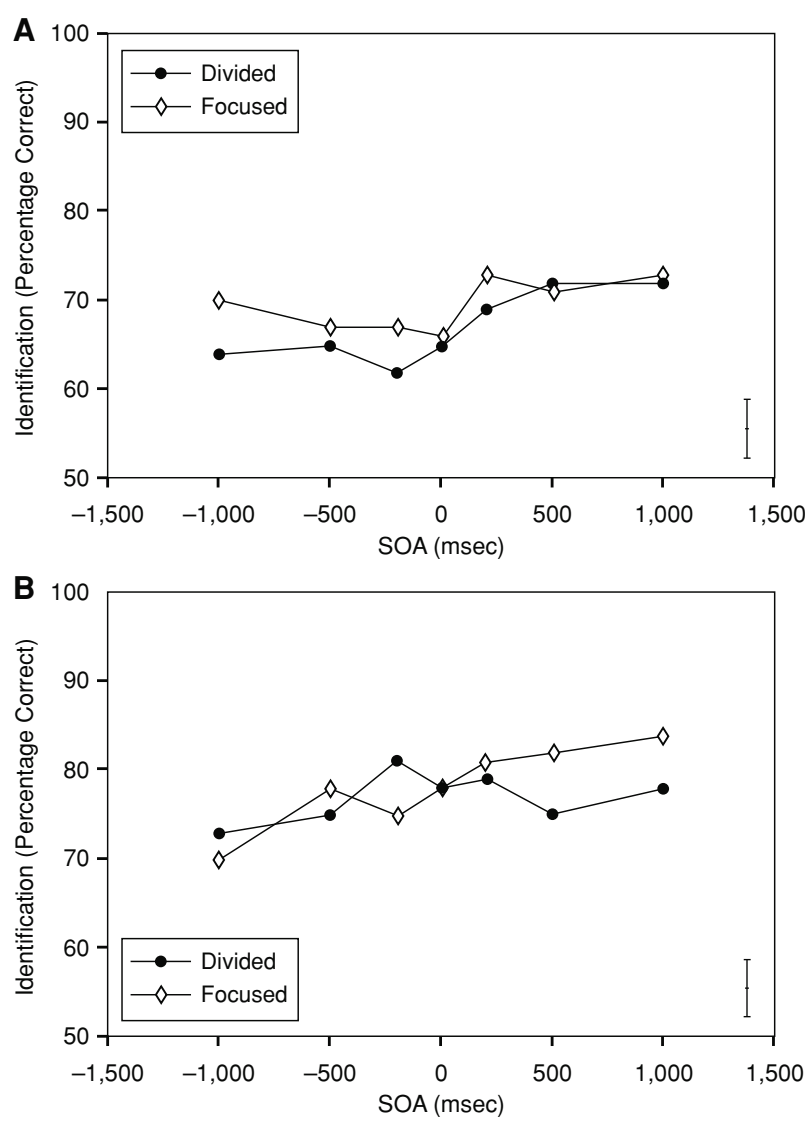

Figure 6. Experiment 4: Group mean percentage of correct responses as a function of condition (focused or divided attention) and stimulus onset asynchrony (SOA). Positive SOAs reflect T2 performance at each SOA after T1. Negative SOAs reflect T1 performance at each SOA backward from T2. (A) Auditory targets. (B) Visual targets. The error bars (bottom right of each panel) show estimates of \pm 1 standard deviation of the mean for each graph point, based on pooled within-subjects error terms.

between auditory and visual targets. Our results suggest that interference time-locked to target identification was unlikely in their study. Instead, some more general factor may have led to reduced performance in divided-attention blocks.

As we have said, there has been some variability in the results of previous attentional blink studies. Some, like ours, have shown no blink in the cross-modal case (Duncan et al., 1997; Soto-Faraco \& Spence, 2002). Some have shown a distinctly reduced blink (Arnell \& Jenkins, 2004; Arnell \& Larson, 2002; Potter et al., 1998, Experiment 5). One previous study even obtained comparable results for within- and cross-modality conditions (Arnell \& Jolicœur, 1999). An interesting question is: Which experimental conditions give rise to cross-modality attentional limitations, and which conditions allow parallel processing across modalities, as in the present study? Taken together, however, the results make clear that a major part of the classical attentional blink arises from a modality-specific processing conflict. Our results show that this holds true for simple discriminations of stimulus intensity as it does for more complex symbol identification.

\section{REFERENCES}

AllPORT, D. A. (1980). Attention and performance. In G. Claxton (Ed.), Cognitive psychology: New directions (pp. 112-153). London: Routledge \& Kegan Paul.

Arnell, K. M., \& Duncan, J. (2002). Separate and shared sources of dual-task cost in stimulus identification and response selection. Cognitive Psychology, 44, 105-147.

Arnell, K. M., \& Jenkins, R. (2004). Revisiting within-modality and cross-modality attentional blinks: Effects of target-distractor similarity. Perception \& Psychophysics, 66, 1147-1161.

Arnell, K. M., \& Jolicceur, P. (1999). The attentional blink across stimulus modalities: Evidence for central processing limitations. Journal of Experimental Psychology: Human Perception \& Performance, 25, 630-648.

Arnell, K. M., \& LARson, J. M. (2002). Cross-modality attentional blinks without preparatory task-set switching. Psychonomic Bulletin \& Review, 9, 497-506.

Bonnel, A.-M., \& HAFTER, E. R. (1998). Divided attention between simultaneous auditory and visual signals. Perception \& Psychophysics, 60, 179-190.

Bourke, P. A., Duncan, J., \& Nimmo-Smith, I. (1996). A general factor involved in dual-task performance decrement. Quarterly Journal of Experimental Psychology, 49A, 525-545.

Broadbent, D. E. (1958). Perception and communication. Oxford: Pergamon.

Chelazzi, L., Duncan, J., Miller, E. K., \& Desimone, R. (1998). Responses of neurons in inferior temporal cortex during memory-guided visual search. Journal of Neurophysiology, 80, 2918-2940.

Desimone, R., \& Duncan, J. (1995). Neural mechanisms of selective visual attention. Annual Review of Neuroscience, 18, 193-222.

Duncan, J. (1984). Selective attention and the organization of visual information. Journal of Experimental Psychology: General, 113, 501-517.

Duncan, J., Martens, S., \& WARD, R. (1997). Restricted attentional capacity within but not between sensory modalities. Nature, 387, 808-810.

JoLICEUR, P. (1999). Restricted attentional capacity between sensory modalities. Psychonomic Bulletin \& Review, 6, 87-92.

Kahneman, D. (1973). Attention and effort. Englewood Cliffs, NJ: Prentice Hall.

LogAn, G. D. (1978). Attention in character-classification tasks: Evidence for the automaticity of component stages. Journal of Experimental Psychology: General, 107, 32-63.

Lu, Z.-L., \& Dosher, B. A. (2000). Spatial attention: Different mechanisms for central and peripheral temporal precues? Journal of Experimental Psychology: Human Perception \& Performance, 26, 15341548

Mondor, T. A. (1998). A transient processing deficit following selection of an auditory target. Psychonomic Bulletin \& Review, 5, 305-311.

Navon, D., \& Gopher, D. (1979). On the economy of the humanprocessing system. Psychological Review, 86, 214-255.

PASHler, H. (1994). Dual-task interference in simple tasks: Data and theory. Psychological Bulletin, 116, 220-244.

PAshler, H., \& BADGio, P. C. (1987). Attentional issues in the identification of alphanumeric characters. In M. Coltheart (Ed.), Attention and performance XII: The psychology of reading (pp. 63-81). Hillsdale, NJ: Erlbaum.

Potter, M. C., Chun, M. M., Banks, B. S., \& Muckenhoupt, M. (1998). Two attentional deficits in serial target search: The visual attentional blink and an amodal task-switch deficit. Journal of Experimental Psychology: Learning, Memory, \& Cognition, 24, 979-992.

RAYMOND, J. E., Shapiro, K. L., \& Arnell, K. M. (1992). Temporary suppression of visual processing in an RSVP task: An attentional 
blink? Journal of Experimental Psychology: Human Perception \& Performance, 124, 849-860.

Reynolds, J. H., Chelazzi, L., \& Desimone, R. (1999). Competitive mechanisms subserve attention in macaque areas V2 and V4. Journal of Neuroscience, 19, 1736-1753.

Soto-Faraco, S., \& SPence, C. (2002). Modality specific auditory and visual temporal processing deficits. Quarterly Journal of Experimental Psychology, 55A, 23-40.
Treisman, A. M., \& Davies, A. (1973). Divided attention to ear and eye. In S. Kornblum (Ed.), Attention and performance IV (pp. 101-117). New York: Academic Press.

(Manuscript received December 1, 2003; revision accepted for publication March 9, 2005.) 